\title{
A plasma SNORD33 signature predicts platinum benefit in metastatic triple-negative breast cancer patients
}

\author{
Biyun Wang ${ }^{1 \dagger}$, Yannan Zhao ${ }^{1,2 \dagger}, \mathrm{Yi} \mathrm{Li}^{1,2 \dagger}{ }^{+}$, Yingying $\mathrm{Xu}^{2 \dagger}$, Yun Chen ${ }^{3}$, Qiuyu Jiang ${ }^{2,4}$, Dingjin Yao ${ }^{2}$, Li Zhang ${ }^{2}$, \\ Xichun $\mathrm{Hu}^{1 *}$, Chaowei Fu ${ }^{3 *}$, Si Zhang ${ }^{2^{*}}$ and She Chen ${ }^{2^{*}}$ (D)
}

Keywords: Breast cancer, Platinum, Prognostic biomarker, SNORD33, MeCP2, Chemoresistance

\begin{abstract}
Main text
Breast cancer is the most common cancer in women [1]. Triple-negative breast cancer (TNBC), defined by the absence of estrogen receptor (ER), progesterone receptor (PR) and ErbB2 receptor (HER2), accounts for $10-20 \%$ of all invasive breast cancers [2]. TNBC has limited treatment options, is prone to recurrence and metastasis, and has a poor prognosis [3]. Platinum has been proved efficacious and is widely used in the neoadjuvant and metastatic treatment of TNBC [4]. However, many patients are refractory to platinum-based regimens and quickly relapse. Here, we aimed to identify a reliable, robust and convenient liquid biopsy approach for rapid prediction of platinum response in TNBC patients.

First, we generated a cisplatin (DDP)-resistant TNBC cell line, MDA-MB-231/DDP, which is 5-fold more resistant to cisplatin than its parental line MDA-MB-231
\end{abstract}

\footnotetext{
*Correspondence: xchu2009@hotmail.com; fcw@fudan.edu.cn; zhangsi@fudan.edu.cn; shechen@fudan.edu.cn

†Biyun Wang, Yannan Zhao, Yi Li and Yingying Xu contributed equally to this work.

1 Department of Medical Oncology, Fudan University Shanghai Cancer Center, Shanghai Medical College, Fudan University, 270 Dong'an Road, Xuhui District, Shanghai 200032, China

${ }^{2} \mathrm{NHC}$ Key Laboratory of Glycoconjugate Research, Department of Biochemistry and Molecular Biology, School of Basic Medical Sciences, Fudan University, 130 Dong'an Road, Xuhui District, Shanghai 200032,

China

${ }^{3}$ School of Public Health, Key Laboratory of Public Health Safety, NHC Key Laboratory of Health Technology Assessment, Fudan University, Shanghai, China

Full list of author information is available at the end of the article
}

(estimated IC50 values: $39.6 \mu \mathrm{mol} / \mathrm{L}$ versus $7.93 \mu \mathrm{mol} / \mathrm{L}$ ) (Fig. 1a). A total of 277 RNA were identified to be aberrantly expressed in 231/DDP cells using RNA-seq analyses (Fig. 1c and S1a). Interestingly, small nucleolar RNAs (snoRNAs) showed the highest variation rate (12.3\%), compared with other groups, such as mRNA, lncRNAs and miRNAs (Fig. S1a, right panel). snoRNAs are noncoding RNA (ncRNA) molecules of 60-300 nucleotides in length and can be detected in plasma or serum [5]. The amount of snoRNAs in blood indicates different disease status and associates with clinicopathological features and prognosis [6-9]. Compared with miRNA, ctDNA and exosome, snoRNAs are more stable, technically easy to enrich and detect, therefore, more suitable to serve as prognostic biomarkers [7, 10-13]. We then validated the expression of snoRNAs using qRT-PCR. Among all changed snoRNAs, SNORD33 showed the largest fold (32.2) of change (Fig. 1c), and with relative high abundance (Fig. 1b-d). The antisense oligonucleotide (ASO2) successfully silenced SNORD33 expression in MDAMB-231, MDA-MB-468 and SUM149PT cells (Fig. S1b). SNORD33 knockdown reduced cisplatin-induced cell death, as measured by CCK-8 assay (Fig. 1e) and colony formation assay (Fig. S2a). Furthermore, a significantly decreased apoptotic cells (Fig. S2b), along with significantly increased level of anti-apoptotic proteins (Mcl-1 and Bcl-2) and reduced expression of pro-apoptotic proteins (cleaved caspase- 3 and caspase-9), were observed in SNORD33 knockdown cells upon cisplatin treatment

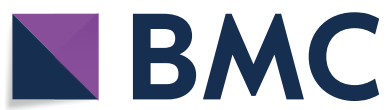

(c) The Author(s) 2022. Open Access This article is licensed under a Creative Commons Attribution 4.0 International License, which permits use, sharing, adaptation, distribution and reproduction in any medium or format, as long as you give appropriate credit to the original author(s) and the source, provide a link to the Creative Commons licence, and indicate if changes were made. The images or other third party material in this article are included in the article's Creative Commons licence, unless indicated otherwise in a credit line to the material. If material is not included in the article's Creative Commons licence and your intended use is not permitted by statutory regulation or exceeds the permitted use, you will need to obtain permission directly from the copyright holder. To view a copy of this licence, visit http://creativecommons.org/licenses/by/4.0/. The Creative Commons Public Domain Dedication waiver (http://creativeco mmons.org/publicdomain/zero/1.0/) applies to the data made available in this article, unless otherwise stated in a credit line to the data. 
(Fig. S2c, d), indicating that reduced SNORD33 promotes cisplatin resistance in TNBC cells.

Considering the potential of SNORD33 as a liquid biopsy biomarker, we investigated the association between the baseline plasma expression of SNORD33 and corresponding prognosis in mTNBC (metastatic triple-negative breast cancer) patients who received firstline platinum-based chemotherapy (Fig. 1f). In the training cohort, 81 patients were divided into high-SNORD33 and low-SNORD33 expression groups according to the median $2-\Delta \Delta$ Ct value of SNORD33 expression in baseline plasma. Baseline characteristics were well-balanced between these two groups (Table S1). As shown in Fig. 1g, low-SNORD33 group had significantly shorter progression-free survival (PFS) than high-SNORD33 group (6.6 versus 10.1 months; $P=0.005$ ). Consistently, in both validation cohort of 128 samples (Fig. 1h) and the combined cohort (Fig. S3a), low-SNORD33 group showed significant worse PFS compared with high-SNORD33 group (validation cohort: 6.5 versus 10.1 months; $P<0.001$; combined cohorts: 6.6 versus 10.1 months; $P<0.001$ ). Likewise, low-SNORD33 group showed significantly reduced overall survival (OS) compared with highSNORD33 group (22.2 versus 40.6 months; $P=0.016$, Fig. S3b) in the combined cohort. Univariate Cox regression analysis showed that low SNORD33 expression, number of metastatic sites, visceral metastasis and liver metastasis were significantly associated with reduced PFS and OS in the combined cohort (Table S2). In the multivariate analysis, the signature of SNORD33 was an independent predictive factor of the PFS with platinum-based chemotherapy in the combined cohort (Table S2). Plasma SNORD33 was significantly higher in patients reaching clinical benefit (CB) (defined as complete response (CR), partial response $(\mathrm{PR})$ and stable disease $(\mathrm{SD})>6$ months) compared with those who failed to reach $\mathrm{CB}$ in the training cohort (11.88 versus $10.98, P=0.012)$, validation group (11.72 versus 11.23, $P=0.006)$ and combined cohorts (11.88 versus $10.98, P=0.038$ ) (Fig. 1j). Furthermore, Kaplan-Meier survival curves from patients treated with non-platinum chemotherapy (gemcitabine plus paclitaxel) (Table S3) revealed that variation in baseline plasma SNORD33 profile did not associate with PFS (5.5 versus 6.5 months; $P=0.535$, Fig. 1i), indicating that the predictive value of SNORD33 expression is platinum-specific. BRCA mutation status, the most studied biomarker for platinum sensitivity in breast cancer, did not correlate with plasma SNORD33 level (Fig. 1k), nor related to PFS in patients with platinum-based chemotherapy (Fig. 11).

ROC test was used to evaluate the value of SNORD33 in predicting PFS in $\mathrm{mTNBC}$ patients treated with platinum-based regimens in the combined cohort. SNORD33 signature showed the strongest predictive value $(\mathrm{AUC}=0.652$ ) for PFS, compared with other single clinicopathological risk factors (liver metastasis,

\footnotetext{
(See figure on next page.)

Fig. 1 Reduced SNORD33 level predicts cisplatin (DDP) resistance in mTNBC. a 231/DDP cells are resistant to cisplatin. 231 and 231/DDP cells were incubated with indicated concentration of DDP for $48 \mathrm{~h}$. CCK8 assays were performed to detect cell viability. $n=3$; ${ }^{* * *}$ represents $P<0.001$; two-tailed t-test. $\mathbf{b}$ Heat map analysis displays the differential expression of snoRNAs including SNORD33 in 231 and 231/DDP cells. c Volcano map analysis shows that SNORD33 expression is most significantly reduced in 231/DDP cells. d SNORD33 is down-regulated in 231/DDP cells. SNORD33 levels in normal 231 cells and cisplatin-resistant 231/DDP cells were assessed by qRT-PCR and normalized against U6. $n=3$; ** represents $P<0.01$; two-tailed t test. e SNORD33 knockdown increases cell viability of cisplatin treated TNBC cells. SNORD33 was knocked down in MDA-MB-231, MDA-MB-468 and SUM149PT cells. Cells were then treated with cisplatin at indicated concentrations for $48 \mathrm{~h}$ and cell viability was determined by CCK8 assay. $n=3$; ** represents $P<0.01$, ${ }^{* * *}$ represents $P<0.001$; two-tailed t test. f Patients selection and study design: plasma from patients $(n=209)$ who received platinum-based regimens in NCT01287624, NCT02341911 and NCT02546934 trials were included in platinum-regimen cohort and those $(n=45)$ who received non-platinum regimen were included in non-platinum-regimen cohort. Peripheral blood mononuclear cells from patients $(n=114)$ in platinum-regimen cohort were randomly tested for gBRCA mutation. $\mathbf{g}$, $\mathbf{h}$ Kaplan-Meier survival curves for median progression-free survival (PFS) in mTNBC patients receiving first-line platinum-based regimen based on the expression of SNORD33. Training cohort $(n=81), P=0.005$ (g); validation cohort $(n=128), P<0.001$ (h). Cut-off threshold was median value in these cohort; log-rank test. i Kaplan-Meier survival curves for median PFS in MTNBC patients received first-line non-platinum-based regimen. Cut-off threshold was median value in this cohort, $P=0.053$; log-rank test. $\mathbf{j}$ Plasma SNORD33 level was significantly higher in patients reaching clinical benefit (CB, $C R+P R+S D>6$ months). Training cohort (11.88 versus $10.98, P=0.012$ ), validation group (11.72 versus $11.23, P=0.006)$, combined cohorts (11.88 versus $10.98, P=0.038)$; two-tailed t test. $\mathbf{k}$, I BRCA mutation neither correlates with plasma SNORD33 level (1.27 versus $1.22, P=0.949)(\mathbf{k})$, nor relates to PFS (I) in patients with platinum-based chemotherapy. Kaplan-Meier survival curves for median PFS in mTNBC patients received first-line non-platinum-based regimen based on gBRCA mutation (I). Cut-off threshold was median value in this cohort $(n=114)$. $P=0.961$; log-rank test. $\mathbf{m}$ The prognostic accuracy for platinum response by the SNORD33 signature, liver metastasis and number of metastatic sites. $\mathbf{n}$ Prognostic nomogram to assign the probability of PFS for TNBC patients after first-line platinum treatment initiation. The probability of PFS at 2, 4, 6, 10,12 and 18 months can be obtained as function of total points calculated as the sum points of each specific variable. Points are assigned for each risk factor by drawing a line upward from the corresponding values to the 'point'line. The total sum of points for three risk factors is plotted on the 'total points'line. A vertical line is drawn for reading the corresponding predictions of 2, 4, 6, 10,12 and 18 months PFS probability. o A calibration curve of the nomogram. The calibration of the prediction model was performed by a visual calibration plot comparing the predicted and actual probability of PFS. The 1000 bootstrap resamples for internal validation was used to assess the predictive accuracies of nomogram
} 


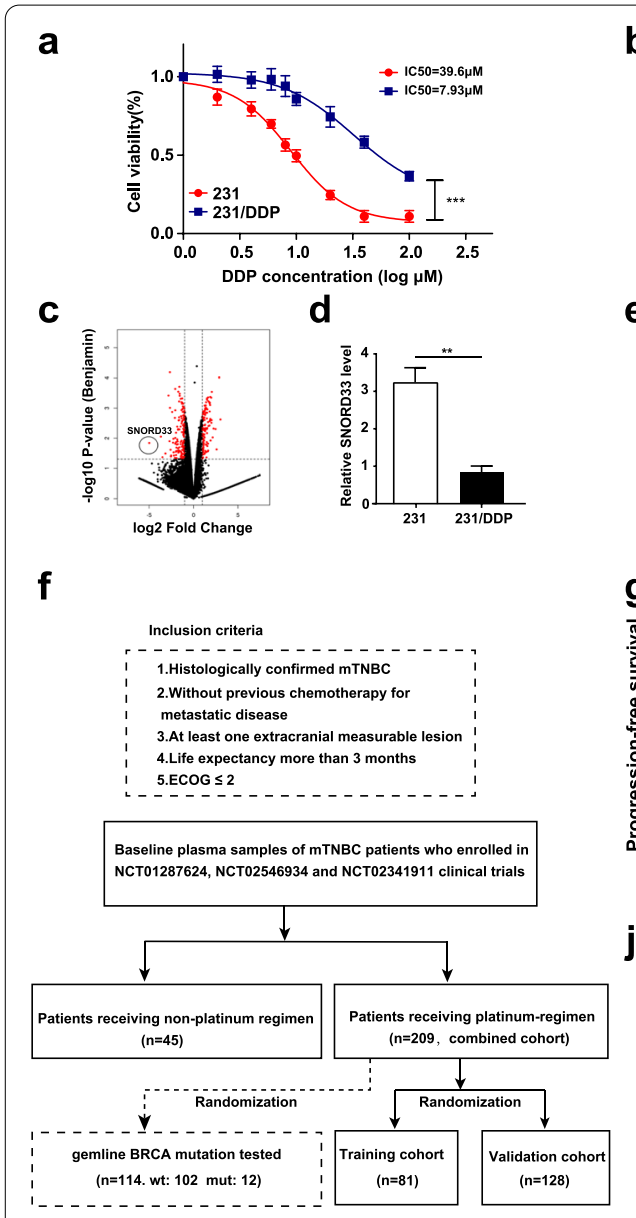

m

Prediction ability of SNORD33 and other clinicopathological factors

\begin{tabular}{|c|c|c|c|}
\hline Factor & AUC & Sensitivity & Specificity \\
\hline SNORD33 & 0.652 & 0.630 & 0.674 \\
\hline Liver metastasis & 0.575 & 0.280 & 0.870 \\
\hline Number of metastatic sites & 0.365 & 0.700 & 0.478 \\
\hline
\end{tabular}

$\mathbf{0}$

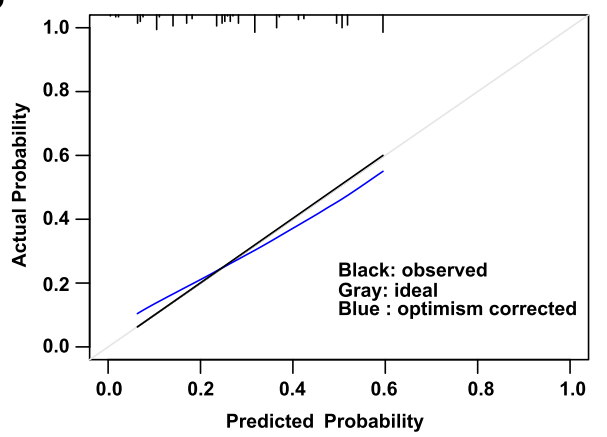

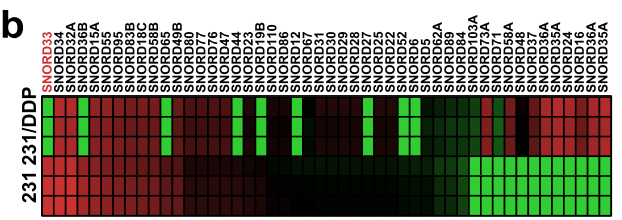

C/D box snoRNAs

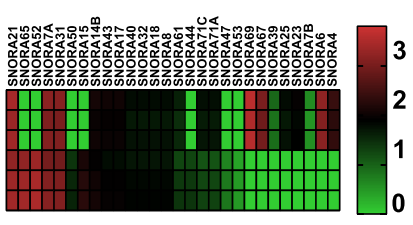

H/ACA box SnoRNAs
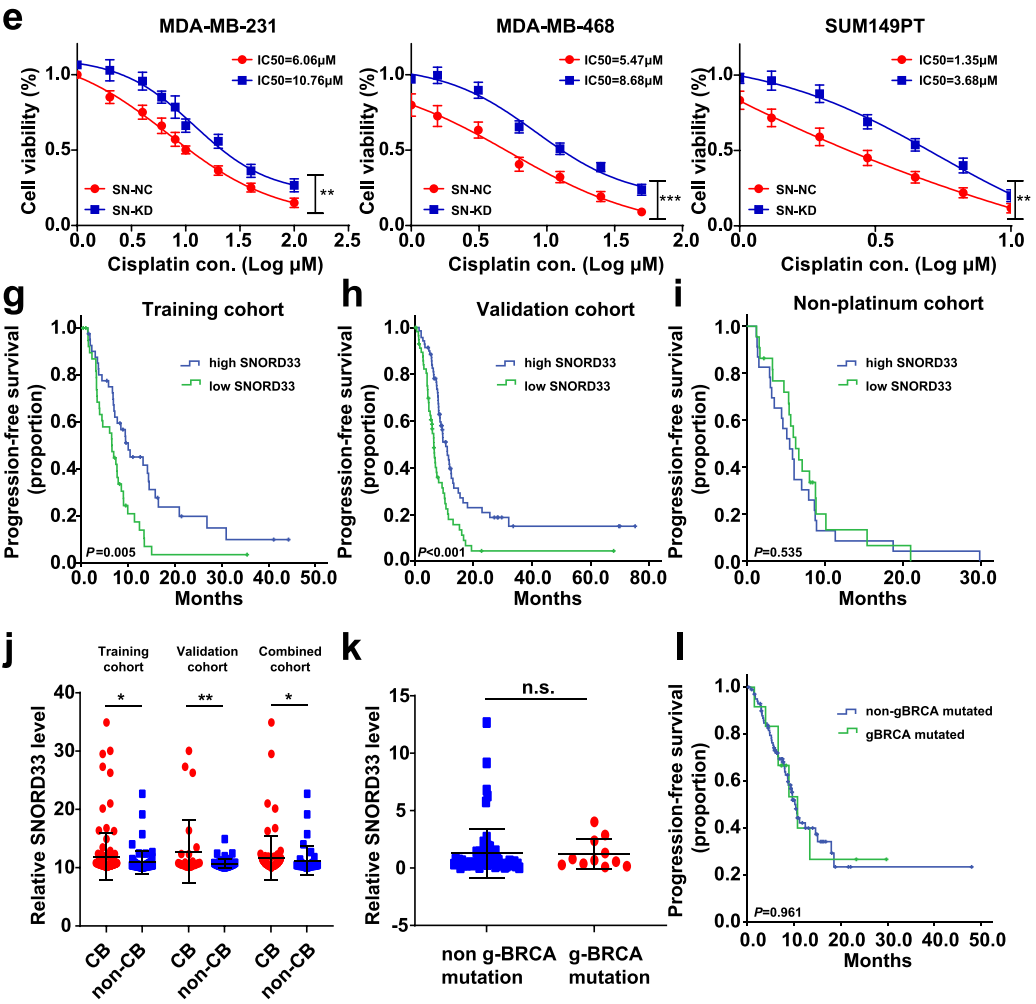

\section{n}

\section{Points}

Number of metastatic sites

SNORD33 expression

Total Points

Linear Predictor

2-months PFS Probability

4-months PFS Probability

6-months PFS Probability

8-months PFS Probability

10-months PFS Probability

12-months PFS Probability

18-months PFS Probability

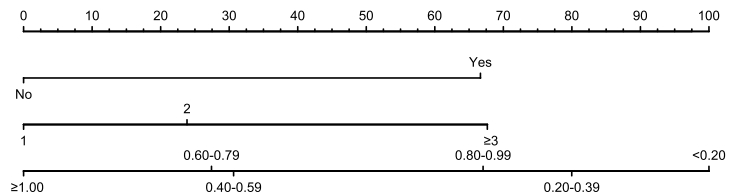

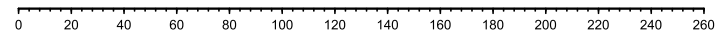
$\begin{array}{llllllllllllll}-1 & -0.8 & -0.6 & -0.4 & -0.2 & 0 & 0.2 & 0.4 & 0.6 & 0.8 & 1 & 1.2 & 1.4 & 1.6\end{array}$

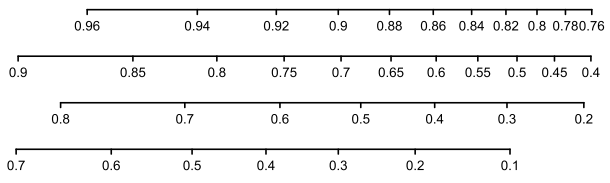

$\begin{array}{lllllllllllll}1 & 1 & 1 & 1 & 1 & 1 & 1 & 1 & 1 & 1 & 1 & 1 & 1 \\ 0.65 & 0.6 & 0.55 & 0.5 & 0.45 & 0.4 & 0.35 & 0.3 & 0.25 & 0.2 & 0.15 & 0.1 & 0.05\end{array}$

$\begin{array}{llllllllllll}0.6 & 0.55 & 0.5 & 0.45 & 0.4 & 0.35 & 0.3 & 0.25 & 0.2 & 0.15 & 0.1 & 0.05\end{array}$

$\begin{array}{llllllll}0.4 & 0.35 & 0.3 & 0.25 & 0.2 & 0.15 & 0.1 & 0.05\end{array}$

Fig. 1 (See legend on previous page.) 
$\mathrm{AUC}=0.575$; number of metastatic sites, $\mathrm{AUC}=0.365$ ), suggesting better performance of SNORD33 signature as a surrogate predictor for platinum-based chemotherapy outcome (Fig. 1m). Based on the estimated coefficients in the multivariate Cox regression model included SNORD33 expression, liver metastasis and number of metastatic sites, we then built a prognostic nomogram to assign PFS probability at 2, 4, 6, 10, 12 and 18 months after first-line platinum treatment. In this nomogram, the point system work by ranking the regression coefficients, with SNORD33 expression converted into 100 points, followed by number of metastatic sites and liver metastasis (yes/no). The final PFS probability was calculated by adding up the score of each item using the nomogram depicted in Fig. 1n. The prediction performance of the nomogram was assessed by the discrimination and calibration. The concordance index ( $\mathrm{C}$ index), estimating the discriminative ability of the nomogram, was 0.664 . A calibration curve also showed high consistency between the predicted and observed PFS probability (Fig. 1o). This nomogram can predict PFS possibility upon platinumbased treatment in different months, information that could be factored in during regimen selection.

Furthermore, a decrease of cisplatin-induced cell toxicity was observed in SNORD33 knocked-down lung adenocarcinoma and urinary bladder carcinoma cell lines (Fig. S4). In 50 metastatic NSCLC patients treated with first-line cisplatin/carboplatin-based regimens, low baseline plasma SNORD33 group had significantly shorter PFS (7.7 versus 15.5 months; $P=0.023)$ than high-SNORD33 group (Table S4, Fig. S5a). And plasma SNORD33 was significantly higher in patients reaching $\mathrm{CB}$ compared with those who failed to reach $\mathrm{CB}$
(1.36 versus $0.34, P=048$, Fig. S5b). The signature of SNORD33 was an independent predictive factor of the PFS with first-line cisplatin/carboplatin-based regimens in lung cancer (Table S5). These data suggest SNORD33 might serve as a feasible predicting biomarker for platinum.

We next investigated the mechanisms underlying SNORD33 affected platinum sensitivity. SNORD33 locates in intron 4 of host gene ribosomal protein L13a (RPL13A) [14]. It participates in the 2- $\mathrm{O}^{\prime}$ - ribose methylation of $18 \mathrm{~S}$ rRNA [14]. Does SNORD33 regulate cisplatin resistance by i) its host gene; ii) sliced into functional miRNA; iii) rRNA modification and subsequent protein translation? We knocked down RPL13A (host gene), DICER enzyme (an endoribonuclease), or SNORD32a (homologue to SNORD33 modifying 18S rRNA during cell proliferation) in MDA-MB-231 cells (Fig. 2a), and found that the cell viability in response to platinum was not changed (Fig. 2b). We then employed RNA-pull down assay combined with mass spectrometry to identify SNORD33 binding proteins. Methyl-CpG-binding protein 2 (MeCP2), an important member of the methylCpG-binding domain (MBD) family with epigenetic control functions [15], was identified as a potential target to convey SNORD33 drug resistance (Fig. 2c, d and Fig. S6a). The interaction between SNORD33 and MeCP2 was further confirmed by RNA immunoprecipitation (RIP) assays (Fig. 2d). The knockdown of SNORD33 in MDA-MB-231 cells did not alter mRNA and protein levels of MeCP2 (Fig. 2e), but reduced mRNA levels of most MeCP2 target genes, including GADD45 $\alpha$, $M Y O D 1$, and CLDN6, which promotes cell apoptosis (Fig. 2f). MeCP2 can repress target-gene expression by

\footnotetext{
(See figure on next page.)

Fig. 2 MeCP2 contributes to SNORD33 mediated cisplatin resistance in mTNBC. aRPL13A, DICER enzyme, or SNORD32a were knocked down in MDA-MB-231 cells. bRPL13A, DICER enzyme, or SNORD32a knockdown do not change the cell viability in response to platinum. These results indicated that platinum resistance arising from loss of SNORD33 may be not caused by alterations of host gene, forming functional miRNA or rRNA modification. $\mathbf{c}$ The silver-stained bands showed the bands of the sense and antisense strands. The different bands were concentrated in the range of 50-80 kDa and 30-40 kDa. Mass spectrometry show that MeCP2 is a candidate protein for SNORD33 interaction. $\mathbf{d}$ MeCP2 immunoprecipitates were enriched by SNORD33. RNA pull-down (upper panel) and RIP (RNA immunoprecipitation) assays (lower panel) were performed in MDA-MB-231 cells. Anti-lgG was used as a negative control. $n=3$; ${ }^{* * *}$ represents $P<0.001$; two-tailed t test. e SNORD33 knockdown in MDA-MB-231 cells doesn't alter MeCP2 mRNA and protein levels. $\mathbf{f}$ mRNA levels of MeCP2 target genes are decreased in MDA-MB-231 cells knocking down of SNORD33. The mRNA levels of GADD45a, MYOD1, FOXF1, CDKL5, CLDN6 in MDA-MB-231 cells were determined by qRT-PCR. $n=3$; ${ }^{*}$ represents $P<0.05$, ${ }^{* *}$ represents $P<0.01$, *** represents $P<0.001$; two-tailed t test. g SNORD33 knockdown promotes MeCP2 binding to downstream target gene promoter. SNORD33 was knocked down in MDA-MB-231 cells and chromatin immunoprecipitation (ChIP) assay was performed. qRT-PCR quantification of the immunoprecipitated DNA were measured. Normal rabbit lgG were used as a negative control. Values represented enrichment relative to input DNA. Data are presented as mean \pm SD; ${ }^{* *}$ represents $P<0.01$, *** represents $P<0.001$; one-way ANOVA. $\mathbf{h}$, i SNORD33 knockdown does not change the binding of co-repressor mSIN3A and HDAC1 to MeCP2. SNORD33 was knocked down in MDA-MB-231 cells and co-immunoprecipitation was performed by MeCP2 antibody. MeCP2, mSIN3A and HDAC1 proteins were detected with western blot (h). The binding affinity between MeCP2/mSIN3A and MeCP2/HDAC1 was quantified (i). $n=3$; n.s. represents not significant; two-tailed t test. $\mathbf{j}$ Down-regulation of MeCP2 rescues SNORD33 knockdown induced cell death. The proliferation of cisplatin treated MDA-MB-231, MDA-MB-468, SUM149PT cells was determined by CCK8 assay. $n=3$; *** represents $P<0.001$; two-tailed t test. $\mathbf{k}$, I Down-regulation of MeCP2 rescues SNORD33 knockdown decreased cell apoptosis ( $\mathbf{k}$ ) and induced alteration of apoptotic markers (I). Apoptosis of cisplatin treated cells was determined by flow cytometric analysis. Western blot was used to detect the indicated apoptotic markers in cisplatin treated cells. $10 \mu \mathrm{M}$ cisplatin for MDA-MB-231, $8 \mu \mathrm{M}$ cisplatin for MDA-MB-468 and $3 \mu \mathrm{M}$ cisplatin for SUM149PT cells
} 


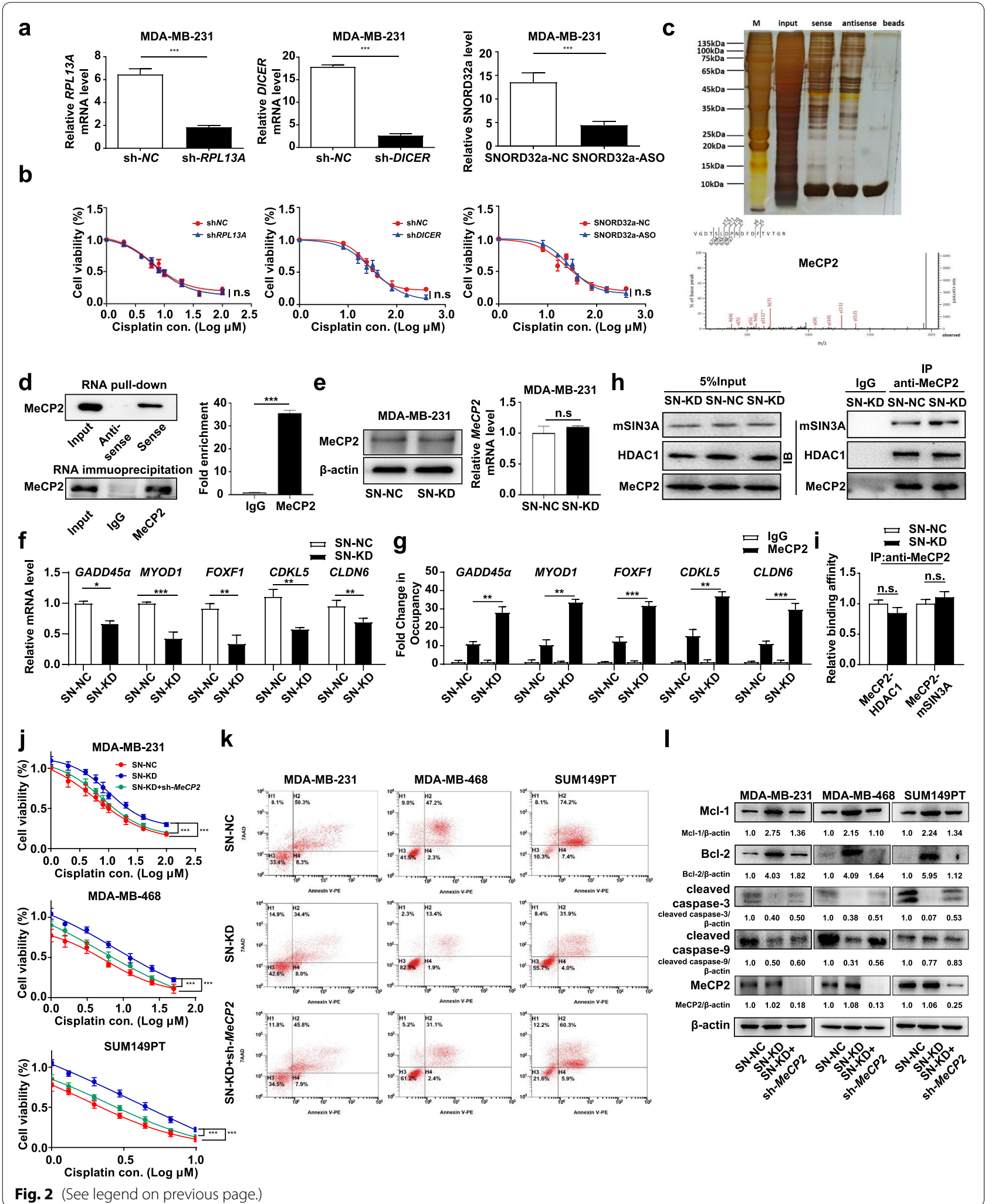


binding to CpG-methyl groups on DNA via its MBD domain [16]. In another model, $\mathrm{MeCP} 2$, via recruiting mSIN3A and HDAC1, induces histone deacetylation, chromatin compaction, resulting in gene repression [16] (Fig. S6b). We observed that SNORD33 knockdown promoted MeCP2 binding to target gene promoter in MDAMB-231 cells by chromatin immunoprecipitation (ChIP) assay (Fig. $2 \mathrm{~g}$ ), while the formation of MeCP2/mSIN3A/ HDAC1 complex was not affected (Fig. 2h-i). Therefore, SNORD33 may regulate MeCP2 targeted gene expression through disrupting its binding with methylated CpG DNA (Fig. S6b). We then knocked down $M e C P 2$ in SNORD33-knockdown MDA-MB-231, MDA-MB-468 and SUM149PT cell lines (Fig. S6c). Down-regulation of MeCP2 expression can partially reverse the platinum resistance of TNBC cell lines caused by loss of SNORD33, as measured by CCK-8 assay (Fig. 2j) and colony formation assay (Fig. S7). Consistently, MeCP2 down-regulation attenuated SNORD33-knockdown induced decreased apoptotic cells and pro-apoptotic proteins, and increased anti-apoptotic proteins (Fig. 2k, 1). Together, these data imply that $\mathrm{MeCP} 2$ contributes to SNORD33 mediated platinum resistance in TNBC cells.

\section{Conclusions}

Together, we identified plasma SNORD33 signature as a predictor for the sensitivity of platinum-based chemotherapy in mTNBC patients. Furthermore, we showed that MeCP2 could convey SNORD33 associated platinum resistance. Our findings may have immediate translational relevance for precision platinum-based chemotherapy.

\begin{abstract}
Abbreviations
ASO: Antisense oligonucleotides; AUC: Area under the curve; BCl-2: B-cell lymphoma-2; Cl: Confidence interval; CLDN6: Claudin 6; ER: Estrogen receptor; GADD45a: Growth arrest and DNA damage inducible alpha; HER2: ErbB2 receptor; HR: Hazard ratio; MBD: Methyl-CpG-binding domain; MCl-I: Myeloid cell leukemia-1; MeCP2: Methyl-CpG-binding protein 2; mTNBC: Metastatic triple negative breast cancer; MYOD1: Myogenic differentiation 1; ncRNA: Noncoding RNA; OS: Overall survival; PFS: Progress-free survival; PR: Progesterone receptor; RIP: RNA immunoprecipitation; ROC: Receiver operating characteristic; snoRNAs: Small nucleolar RNAs; TNBC: Triple negative breast cancer.
\end{abstract}

\section{Supplementary Information}

The online version contains supplementary material available at https://doi. org/10.1186/s12943-022-01504-0.

\section{Additional file 1. Methods.}

Additional file 2: Supplementary Figure 1. Aberrantly expressing RNA in 231/DDP cells. Supplementary Figure 2. SNORD33 knockdown increases proliferation and decreases apoptosis of TNBC cells. Supplementary Figure 3. Reduced SNORD33 level is correlated with poor prognosis of mTNBC patients who received first-line platinum-based chemotherapy. Supplementary Figure 4. Increased cell viability was observed in cisplatin treated cell lines with SNORD33 knockdown. Supplementary Figure 5. Reduced SNORD33 level correlates with poor prognosis of non-small cell lung cancer (NSCLC) patients who received first-line platinum-based chemotherapy. Supplementary Figure 6. MeCP2 is a candidate protein binding with SNORD33. Supplementary Figure 7. Down-regulation of MeCP2 partially rescues SNORD33 knockdown increased cell colony formation. Supplementary Figure 8. Down-regulation of MeCP2 rescues SNORD33 knockdown decreased cell apoptosis and induced alteration of apoptotic markers.

Additional file 3: Supplementary Table 1. Baseline characteristics in mTNBC patients received first-line platinum-containing regimens. Supplementary Table 2. Univariate and multivariate analysis of prognostic factors associated with progression-free survival and overall survival in the combined cohort. Supplementary Table 3. Baseline characteristics in $\mathrm{mTNBC}$ patients received first-line non-platinum-containing regimens. Supplementary Table 4. Baseline characteristics in lung adenocarcinoma patients received first-line platinum-containing regimens. Supplementary Table 5. Univariate and multivariate analysis of prognostic factors associated with progression-free survival in lung adenocarcinoma patients.

Additional file 4.

\section{Acknowledgements}

We thank Lina Ma for plasma sample collection and processing.

\section{Authors' contributions}

S.C. conceived the study. S.C., Y.Z. and Y.X. wrote the manuscript, designed and arranged the figures and tables. B.W., Y.Z., Y.L., Y.X., Q.J. and D.Y. performed experiments. Y.Z. and Y.C. performed bioinformatic, statistical analyses of clinical data. Y.Z. and L.Z. scored tissue-microarrays. S.C. and S.Z. supervised the study and data analysis. X.H. provided clinical sample and the related patients' information. C.F. supervised bioinformatic and statistical analyses. All authors read and approved the final version of the manuscript.

Authors' information

Not applicable.

\section{Funding}

This work was supported by the National Natural Science Foundation (81972294, 81874114, 81770137, 81772968, 81772615).

\section{Availability of data and materials}

Original RNA sequencing and mass spectrometry data is available from the corresponding author upon reasonable request.

\section{Declarations}

\section{Ethics approval and consent to participate}

Peripheral blood specimens were obtained from three clinical trials CBCSG006, NCT02546934 and NCT02341911. The collection of blood samples and access to clinical data for research were approved by the Institutional Review Board of the Fudan University Shanghai Cancer Center (FUSCC).

Consent for publication

Not applicable.

\section{Competing interests}

The authors declare no conflict of interest.

\section{Author details}

${ }^{1}$ Department of Medical Oncology, Fudan University Shanghai Cancer Center, Shanghai Medical College, Fudan University, 270 Dong'an Road, Xuhui District, Shanghai 200032, China. ${ }^{2} \mathrm{NHC}$ Key Laboratory of Glycoconjugate Research, Department of Biochemistry and Molecular Biology, School of Basic Medical Sciences, Fudan University, 130 Dong'an Road, Xuhui District, Shanghai 200032 , China. ${ }^{3}$ School of Public Health, Key Laboratory of Public Health Safety, NHC Key Laboratory of Health Technology Assessment, Fudan University, Shanghai, China. ${ }^{4}$ Department of Gastroenterology and Hepatology, Shanghai Institute of Liver Diseases, Zhongshan Hospital, Fudan University, Shanghai, China. 
Received: 2 November 2021 Accepted: 10 January 2022

Published online: 18 January 2022

\section{References}

1. Sung H, Ferlay J, Siegel RL, Laversanne M, Soerjomataram I, Jemal A, et al. Global cancer statistics 2020: GLOBOCAN estimates of incidence and mortality worldwide for 36 cancers in 185 countries. CA Cancer J Clin. 2021;71(3):209-49.

2. Perou CM, Sorlie T, Eisen MB, van de Rijn M, Jeffrey SS, Rees CA, et al. Molecular portraits of human breast tumours. Nature. 2000;406(6797):747-52.

3. Berrada N, Delaloge S, Andre F. Treatment of triple-negative metastatic breast cancer: toward individualized targeted treatments or chemosensitization? Ann Oncol. 2010;21(Suppl 7):vii30-5.

4. Denkert C, Liedtke C, Tutt A, von Minckwitz G. Molecular alterations in triple-negative breast cancer-the road to new treatment strategies. Lancet. 2017;389(10087):2430-42.

5. Bachellerie JP, Cavaille J, Huttenhofer A. The expanding snoRNA world. Biochimie. 2002;84(8):775-90.

6. Cui L, Nakano K, Obchoei S, Setoguchi K, Matsumoto M, Yamamoto T, et al. Small nucleolar noncoding RNA SNORA23, up-regulated in human pancreatic ductal adenocarcinoma, regulates expression of Spectrin repeat-containing nuclear envelope 2 to promote growth and metastasis of xenograft tumors in mice. Gastroenterology. 2017;153(1):292-306 e2.

7. Liao J, Yu L, Mei Y, Guarnera M, Shen J, Li R, et al. Small nucleolar RNA signatures as biomarkers for non-small-cell lung cancer. Mol Cancer. 2010;9:198.

8. Mei YP, Liao JP, Shen J, Yu L, Liu BL, Liu L, et al. Small nucleolar RNA 42 acts as an oncogene in lung tumorigenesis. Oncogene. 2012;31(22):2794-804.

9. Okugawa Y, Toiyama Y, Toden S, Mitoma H, Nagasaka T, Tanaka K, et al. Clinical significance of SNORA42 as an oncogene and a prognostic biomarker in colorectal cancer. Gut. 2017;66(1):107-17.

10. Appaiah HN, Goswami CP, Mina LA, Badve S, Sledge GW Jr, Liu Y, et al. Persistent upregulation of U6:SNORD44 small RNA ratio in the serum of breast cancer patients. Breast Cancer Res. 2011;13(5):R86.

11. Baraniskin A, Nopel-Dunnebacke S, Ahrens M, Jensen SG, Zollner H, Maghnouj A, et al. Circulating U2 small nuclear RNA fragments as a novel diagnostic biomarker for pancreatic and colorectal adenocarcinoma. Int J Cancer. 2013;132(2):E48-57.

12. Crea F, Quagliata L, Michael A, Liu HH, Frumento P, Azad AA, et al. Integrated analysis of the prostate cancer small-nucleolar transcriptome reveals SNORA55 as a driver of prostate cancer progression. Mol Oncol. 2016;10(5):693-703.

13. Tosar JP, Garcia-Silva MR, Cayota A. Circulating SNORD57 rather than piR54265 is a promising biomarker for colorectal cancer: common pitfalls in the study of somatic piRNAs in cancer. RNA. 2021;27(4):403-10.

14. Rimer JM, Lee J, Holley CL, Crowder RJ, Chen DL, Hanson PI, et al. Longrange function of secreted small nucleolar RNAs that direct 2'-O-methylation. J Biol Chem. 2018;293(34):13284-96.

15. Vieira JP, Lopes F, Silva-Fernandes A, Sousa MV, Moura S, Sousa S, et al. Variant Rett syndrome in a girl with a pericentric X-chromosome inversion leading to epigenetic changes and overexpression of the MECP2 gene. Int J Dev Neurosci. 2015;46:82-7.

16. Pandey S, Pruitt K. Functional assessment of MeCP2 in Rett syndrome and cancers of breast, colon, and prostate. Biochem Cell Biol. 2017;95(3):368-78.

\section{Publisher's Note}

Springer Nature remains neutral with regard to jurisdictional claims in published maps and institutional affiliations.

Ready to submit your research? Choose BMC and benefit from:

- fast, convenient online submission

- thorough peer review by experienced researchers in your field

- rapid publication on acceptance

- support for research data, including large and complex data types

- gold Open Access which fosters wider collaboration and increased citations

- maximum visibility for your research: over $100 \mathrm{M}$ website views per year

At $\mathrm{BMC}$, research is always in progress.

Learn more biomedcentral.com/submissions 